\title{
Adaptive Optics for Improved Retinal Surgery and Diagnostics
} M. S. Humayun, S. R. Sadda, C. A. Thompson, S. S.
Olivier, M. W. Kartz

\section{August 21, 2000}

U.S. Department of Energy

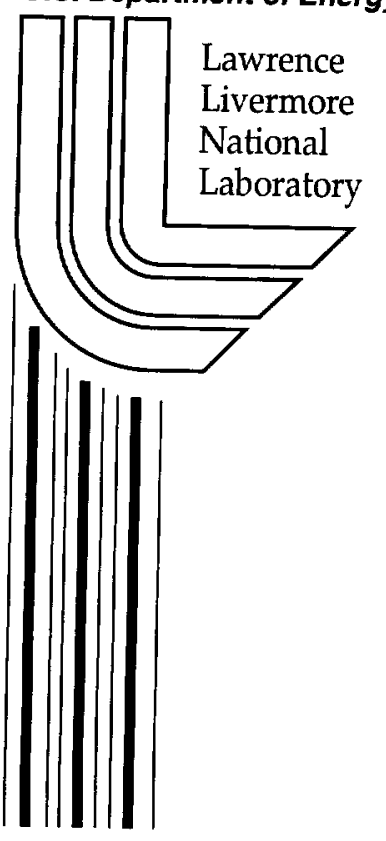




\section{DISCLAIMER}

This document was prepared as an account of work sponsored by an agency of the United States Government. Neither the United States Government nor the University of California nor any of their employees, makes any warranty, express or implied, or assumes any legal liability or responsibility for the accuracy, completeness, or usefulness of any information, apparatus, product, or process disclosed, or represents that its use would not infringe privately owned rights. Reference herein to any specific commercial product, process, or service by trade name, trademark, manufacturer, or otherwise, does not necessarily constitute or imply its endorsement, recommendation, or favoring by the United States Government or the University of California. The views and opinions of authors expressed herein do not necessarily state or reflect those of the United States Government or the University of California, and shall not be used for advertising or product endorsement purposes.

This work was performed under the auspices of the U.S. Department of Energy by the University of California, Lawrence Livermore National Laboratory under Contract No. W-7405-Eng-48.

This report has been reproduced directly from the best available copy.

Available electronically at http://www.doc.gov/bridge

Available for a processing fee to U.S. Department of Energy

And its contractors in paper from

U.S. Department of Energy

Office of Scientific and Technical Information

P.O. Box 62

Oak Ridge, TN 37831-0062

Telephone: (865) 576-8401

Facsimile: (865) 576-5728

E-mail: reports@adonis.osti.gov

Available for the sale to the public from

U.S. Department of Commerce

National Technical Information Service 5285 Port Royal Road

Springfield, VA 22161

Telephone: (800) 553-6847

Facsimile: (703) 605-6900

E-mail: orders@ntis.fedworld.gov

Online ordering: http://www.ntis.gov/ordering.htm

\section{OR}

Lawrence Livermore National Laboratory

Technical Information Department's Digital Library

http://www.llnl.gov/tid/Library.html 


\section{Adaptive Optics for improved retinal surgery and diagnostics}

Mark S. Humayun MD PhD, Srinivas R. Sadda MD

Wilmer Eye Institute. Johns Hopkins University

Charles A. Thompson PhD, Scot S. Olivier PhD, Michael W. Kartz

Lawrence Livermore National Laboratory

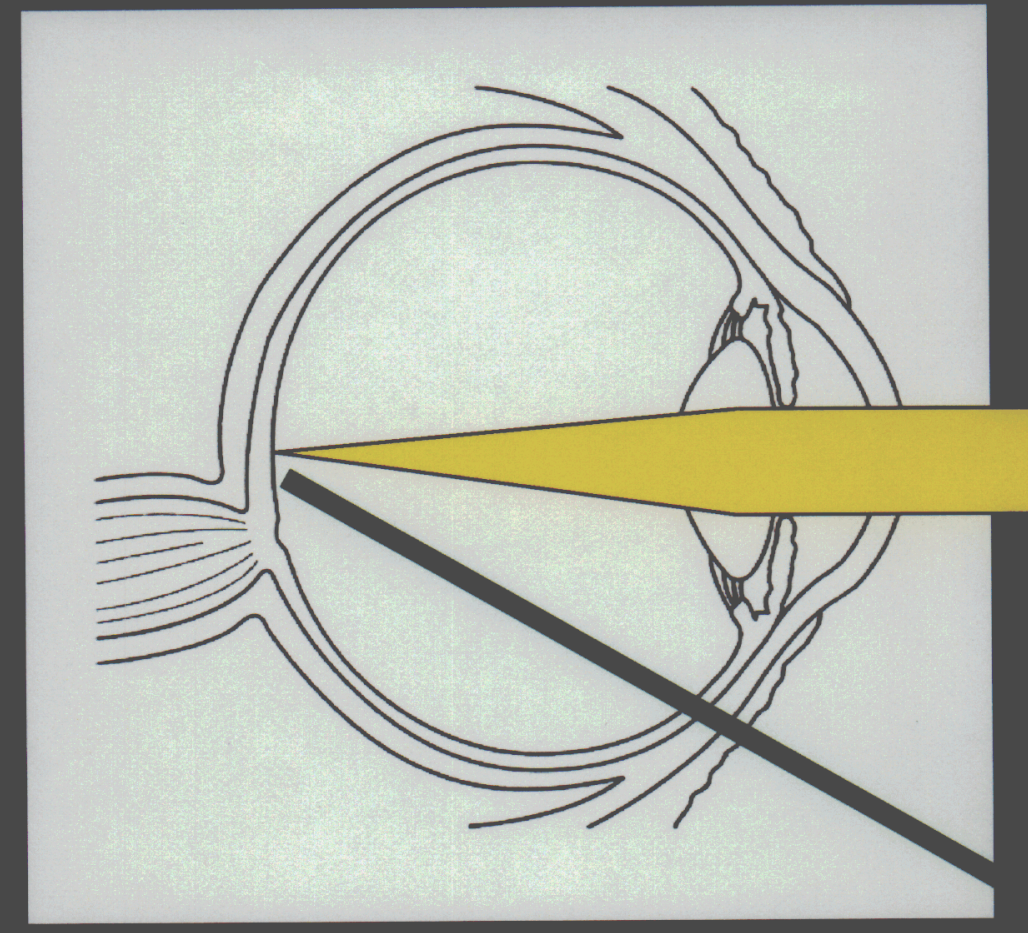

The Wilmer Eye Institute
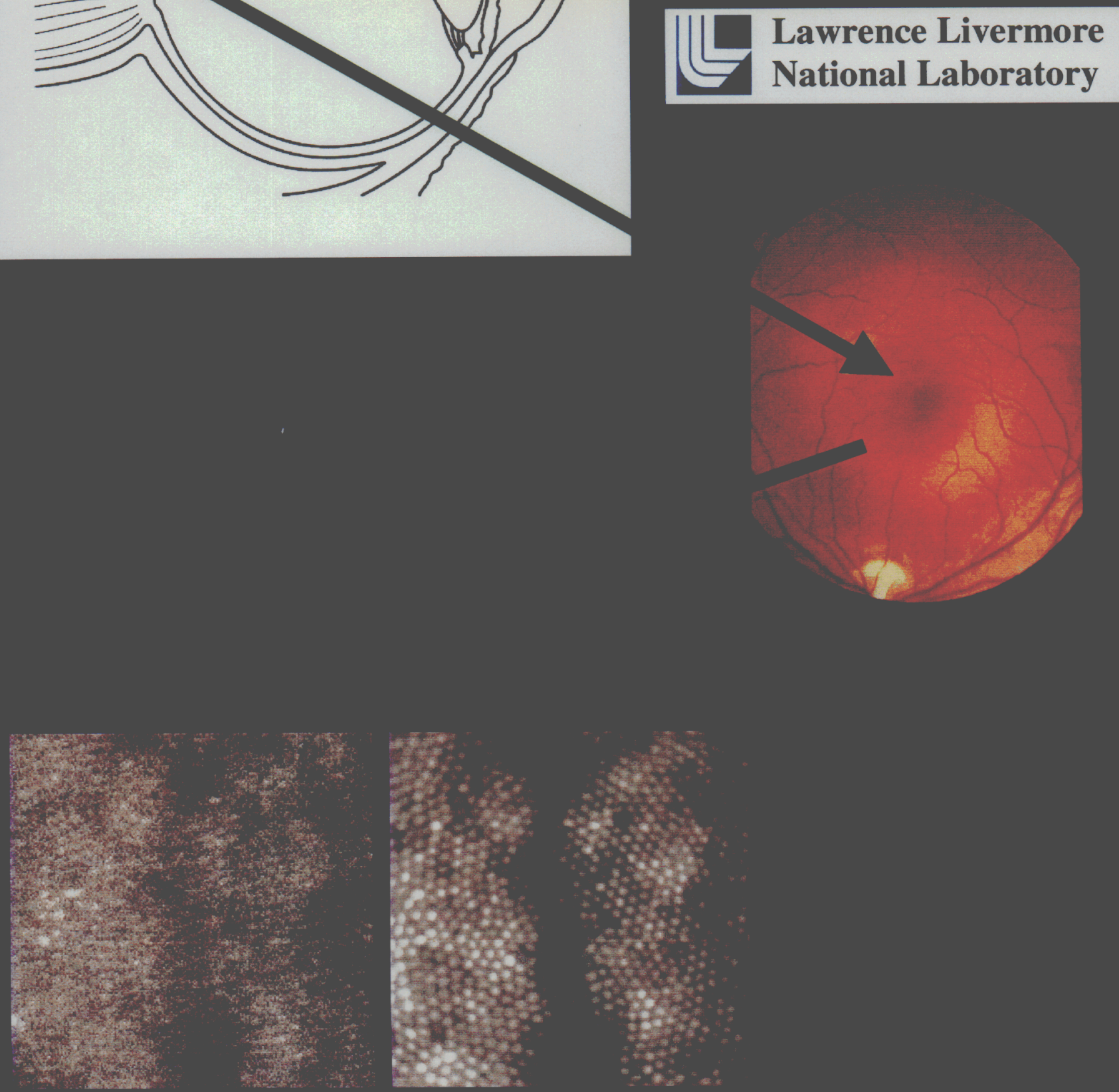


\title{
Adaptive Optics for improved retinal surgery and diagnostics
}

\author{
Mark S. Humayun MD PhD, Srinivas R. Sadda MD \\ Wilmer Eye Institute, Johns Hopkins University \\ Charles A. Thompson PhD, Scot S. Olivier PhD, Michael W. Kartz \\ Lawrence Livermore National Laboratory
}

\begin{abstract}
SUMMARY
It is now possible to field a compact adaptive optics (AO) system on a surgical microscope for use in retinal diagnostics and surgery. Recent developments in integrated circuit technology and optical photonics have led to the capablility of building an AO system that is compact and significantly less expensive than traditional AO systems. It is foreseen that such an $\mathrm{AO}$ system can be integrated into a surgical microscope while maintaining a package size of a lunchbox. A prototype device can be developed in a manner that lends itself well to large-scale manufacturing.
\end{abstract}

\section{MOTIVATION AND SCOPE}

The imaging of living retinal structures is typically performed through the cornea and lens of the patient. Therefore, the aberrations of the eye affect the ulimate resolution of the retinal images. Recent work in vision science has quantified and partially corrected eye aberrations in vivo, by borrowing adaptive optics technology developed for astronomy. Using a 37-actuator deformable mirror, a team headed by David Williams at the University of Rochester has successfully resolved individual $5 \mu \mathrm{m}$ cones in a living human eye. The low resolution of the deformable mirror prevents the system from achieving diffraction-limited imaging. The Rochester system was created as a research, proof-of-concept device and was not optimized for size, performance, or cost. It is

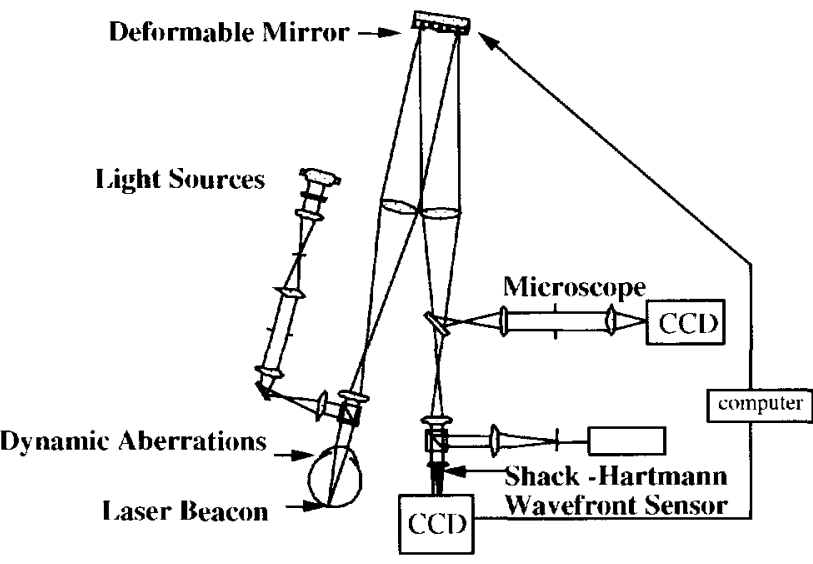

Figure 1. Diagram of and Adaptive Optics system for retinal imaging

based on a traditional 37 actuator deformable glass mirror, which is relatively expensive (approximately $\$ 50,000$ ) and large (about $50 \mathrm{~mm}$ aperture). The resolution limits, coupled with the size and cost, renders this system impractical for most researchers and clinicians from both a functional and financial standpoint.

The AO system operates by illuminating the retina with a point source of laser light (the laser beacon). This beacon reflects back out of the eye and is imaged onto a wavefront sensor where the aberrations of the eye are determined. Once measured, the deformable mirror can be shaped to compensate for these distortions. Improved retinal imaging is then possible by looking back through the system (see microscope location in Figure 1).

It is now feasible to construct an $\mathrm{AO}$ system based on less expensive technology with the resolution necessary to reach diffraction limited imaging. The cost of traditional deformable mirrors typically scales at $\$ 1000$ per actuator and therefore, a system consisting of a modest 100 actuators will cost on the order of $\$ 100,000$. A more feasible approach for a manufactured system would rely on Micro Electro-Mechanical

On the cover, improvements in retinal imaging are made possible through the use of adaptive optics. The image on the bottom left shows a typical microscopic view of a living retina. The image to the right shows improvements in image quality using adaptive optics. Retinal images courtesy of David Williams,

University of Rochester. 


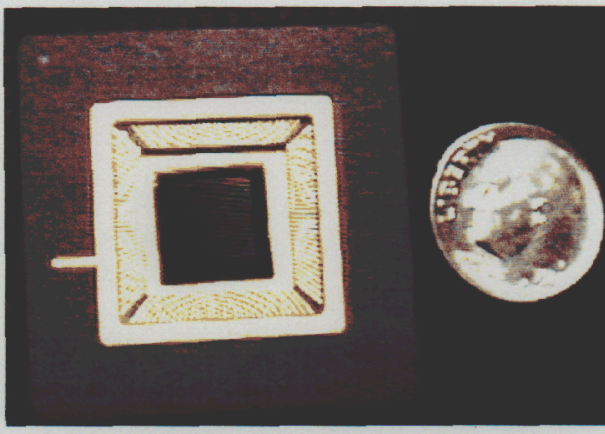

Figure 2. Boston Micromachines

140 actuator $\mu \mathrm{DM}$. systems (MEMs) devices as micro Deformable Mirrors ( $\mu$ DM's). These devices are fabricated using state of the art integrated circuit technology. One device currently available from Boston Micromachines has 140 actuators packaged into a $3.3 \mathrm{~mm} \times 3.3 \mathrm{~mm}$ MEMs mirror. The small size of the device allows for a significant reduction in the package size of the adaptive optics system. These devices lend themselves well to batch fabrication and therefore drastically reduced cost. Today, the 140 actuator $\mu \mathrm{DM}$ mentioned above sells for approximately $\$ 20,000$. Within the next few years, it is estimated that these costs will be reduced by at least an order of magnitude, putting these devices well into the affordable range.

\section{EXPERIENCE}

Over the past two decades, LLNL has developed a core competency in conventional adaptive optics for the control of laser beam quality and the improvement of images made by large ground-based telescopes. More recently, LLNL has been a leader in the development of high-resolution wavefront control capability using new liquid crystal spatial light modulator technology. This new technology has created a major shift in the cost paradigm per wavefront phase control element by four orders of magnitude over conventional adaptive optics. However, liquid crystal technology has several limitations including the speed with which it can adjust wavefront phase, the power-handling capability, and the sensitivity to polarization and temperature changes. These limitations render this technology undesirable for certain applications, but has aided in the continued development of smaller systems with both high-resolution sensing and control of phase distortions maintaining LLNL in the forefront of $\mathrm{AO}$ technology. In order to continue to extend wavefront control capabilities at LLNL, we are beginning to demonstrate an adaptive optics system based on microelectro-mechanical systems (MEMS) spatial light modulator (SLM) technology.

LLNL is currently coordinating a nation-wide consortium through the NSF Center for Adaptive Optics (CfAO) to develop MEMS SLM technology that can be used for wavefront control applications. The CfAO development

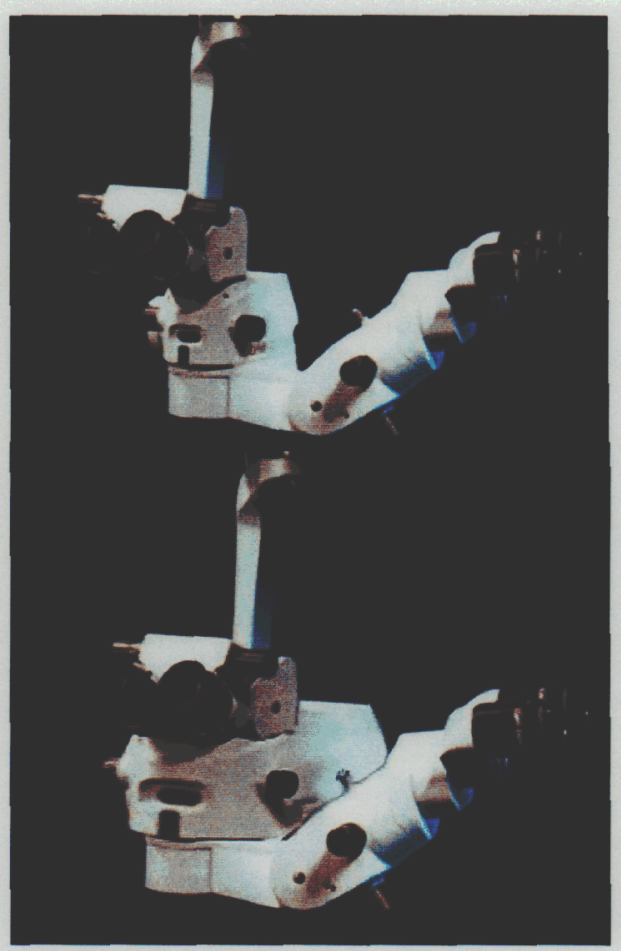

Figure 3. Top - typical ophthalmologic microscope. Bottom- Artist's conception of a microscope modified with an $\mathrm{AO}$ system. plan includes three important risk reduction features. First, there are two parallel development paths based on the most successful current device designs generated at Boston University (BU) and the Air Force Research Laboratory (AFRL). Second, these designs are based on the most established surface micromachining fabrication processes for optical quality devices, at the most established surface micromachining foundries, located at Cronos Integrated Microsystems (CIM) and Sandia National Laboratory (SNL). Third, packaging and drive electronics are being provided by Lucent Technologies based on their extensive investment in these areas for their optical cross-connect switching product, and by Boston Micromachines Corporation (BMC), a start-up company working with Boston University to commercialize their MEMS SLM device technology. 
Vision science is one of key charters of the CfAO and as such LLNL has established a working relationship with David Williams at the University of Rochester. The current LLNL-Rochester collaboration is investigating high resolution adaptive optics for vision science applications. The implementation relies on the modification of the Rochester AO system with a liquid crystal SLM. One of the results of this experimentation will give a quantitative assesment of the wavefront resolution necessary for improved imaging. The experiment, however, is not to miniaturize the Rochester system.

\section{IMPLEMENTATION}

The technical plan for implementation includes consulting with David Williams and staff at the University of Rochester. Williams has been informed about this effort and is interested in the collaboration and possibilities that may arise. His knowledge of ophthalmologic / adaptive optics interaction will be invaluable to our integration efforts. Most of the key ingredients to this prototype exist in a variety of forms either at LLNL or Rochester. Both locations have driver software developed for AO systems that can be modified to use in this prototype, reducing the risks in accurate wavefront sensing and high performance actuator control. One key ingredient to this system is designing the optical and mechanical hardware for small packaging while maintaining research grade optical quality. LLNL maintains a reputation for world class optical design of complex systems, including a long history of adaptive optics development. LLNL interaction with MEMs manufactures will play a key role in the integration of these devices into the stringent packaging requirements necessary for fielding. Although the $\mu \mathrm{DMs}$ are quite small, miniaturization of the packaging and support electronics is ongoing under LLNL coordination.

The intended prototype will be developed as a modification unit to a pre-existing surgical microscope, opening the possibility of partnering with an ophthalmological microscope manufacturer. This partnership can help ease the integration of the AO system onto an exitsting commerical microscope or lead to the development of a completely new model. Some of the components that make up the AO system may exist in a useable form within the Bausch and Lomb family. It is possible that the development of wavefront sensor technology at Technolas may be incorporated into this envisioned AO system.

The estimated timescale for a prototype system is three years. At the end of the first year, a breadboard type arrangement will be set up that incorporates the MEMs device into a working AO system, allowing for software development on a working $\mathrm{AO}$ system. Each of the individual devices that will make up the final integrated system can be tested for functionality before actual integration. Within this first year, the initial optical and mechanical design will be finalized and the components will be ordered. Within the second year, the AO system will be configured onto a working surgical microscope. By the end of the second year, final inspection at LLNL will be performed on the system and it will be delivered to the Wilmer Eye Institute. The Wilmer staff will be trained on the use of the system and their feedback will allow LLNL to improve any software or hardware issues. At the completion of this project, the results of the Wilmer evaluation will be incorporated into a final design that will be ready for commercialization.

\section{CLINICAL APPLICATIONS}

The potential clinical applications for an adaptive optics system for retinal surgery and diagnostics are enormous. The inability to visualize the retinal microarchitecture intraoperatively is currently a major handicap to the further development of vitreoretinal and subretinal surgery.

We can identify several immediate applications for an AO modified surgical microscope: (1) the precise identification of the extent of choroidal neovascularization (CNV) to aid in complete removal of the membrane during submacular surgery; (2) visualization of the retinal microcirculation to facilitate sheathotomy and retinal vein cannulation; and (3) visualization of the internal limiting membrane (ILM) to facilitate removal of epiretinal tissues. These surgeries, which are used for treating age-related macular degeneration (AMD), diabetic retinopathy, complicated retinal detachments, macular puckers, and macular holes, constitute the majority of the procedures currently performed by most vitreo-retinal surgeons. Thus, adaptive optics could markedly improve the current standard of vitreo-retinal surgery. In addition, there are 
numerous potential future applications, particularly for the introduction of therapeutic materials (such as retinal transplants, microspheres of drugs, ) into the subretinal space, or for implanting devices on the retinal surface (such as the retinal microchip/ prosthesis).

Adaptive optics technology would also be invaluable as a diagnostic tool to the retinal surgeon. For example, the detection of preserved photoreceptors over an area of CNV may identify that patient as an individual who could actually benefit from removal or destruction of the CNV. In addition, an AO system allowing imaging of the retinal capillary circulation may obviate the need for fluorescein angiography to assess macular perfusion (e.g in diabetic patients). Moreover, a conventional fluorecein angiogram enhanced with adaptive optics could facilitate and guide the precise laser treatment of CNV. This would be of great importance to patients with AMD, the leading cause of blindness in the United States.

Fundus images captured by AO technology would also be invaluable for following patients and evaluating microscopic changes produced by a variety of therapeutic interventions, proven or experimental. Indeed, ophthalmology (as well as medicine as a whole) has relied heavily on histopathologic correlation to gain insight into the pathophysiology of disease and the effects of various treatments. Adaptive optics presents an avenue by which microscopic/ cellular information may be acquired in an in vivo, non-invasive setting.

To refine the $\mathrm{AO}$ system for clinical application, it is essential that the system be tested in a patient environment. The Wilmer retinal clinical team, which includes Mark Humayun, Eugene de Juan, Michael Cooney, and Srinivas Sadda is willing to evaluate this system in a real patient care setting. 